\title{
Protruding hydrogen atoms as markers for the molecular orientation of a metallocene
}

\author{
Linda Laflör, Michael Reichling and Philipp Rahe*
}

\author{
Full Research Paper \\ Address: \\ Fachbereich Physik, Universität Osnabrück, Barbarastrasse 7, 49076 \\ Osnabrück, Germany \\ Email: \\ Philipp Rahe* - prahe@uni-osnabrueck.de \\ * Corresponding author \\ Keywords: \\ calcium fluoride $\left(\mathrm{CaF}_{2}\right)$; ferrocene; functionalised tips; high-resolution \\ imaging; non-contact atomic force microscopy \\ Beilstein J. Nanotechnol. 2020, 11, 1432-1438. \\ https://doi.org/10.3762/bjnano.11.127 \\ Received: 21 May 2020 \\ Accepted: 14 August 2020 \\ Published: 22 September 2020 \\ Associate Editor: E. Meyer \\ (c) 2020 Laflör et al.; licensee Beilstein-Institut. \\ License and terms: see end of document.
}

\begin{abstract}
A distinct dumbbell shape is observed as the dominant contrast feature in the experimental data when imaging $1,1^{\prime}$-ferrocene dicarboxylic acid (FDCA) molecules on bulk and thin film $\mathrm{CaF}_{2}$ (111) surfaces with non-contact atomic force microscopy (NC-AFM). We use NC-AFM image calculations with the probe particle model to interpret this distinct shape by repulsive interactions between the NC-AFM tip and the top hydrogen atoms of the cyclopentadienyl $(\mathrm{Cp})$ rings. Simulated NC-AFM images show an excellent agreement with experimental constant-height NC-AFM data of FDCA molecules at several tip-sample distances. By measuring this distinct dumbbell shape together with the molecular orientation, a strategy is proposed to determine the conformation of the ferrocene moiety, herein on $\mathrm{CaF}_{2}(111)$ surfaces, by using the protruding hydrogen atoms as markers.
\end{abstract}

\section{Introduction}

It is still a challenge to determine the precise adsorption geometry of three-dimensional (3D) molecules on surfaces. While spectroscopic methods, such as X-ray standing waves [1] or photoelectron diffraction [2], can precisely determine the location of atomic species in ordered molecular systems on surfaces [3], scanning probe methods are commonly employed for the investigation of both ordered and unordered molecular systems as well as of individual and isolated species [4-6]. For example, two different non-planar isomers of dibenzo $[a, h]$ thianthrene molecules could be identified by high-resolution non-contact atomic force microscopy (NC-AFM) [7,8]. Furthermore, the imaging of hydrogen atoms attached to propellane molecules with CO-functionalised tips was suggested to enable the identification of three-dimensional molecules [9]. Very recently, a combination of NC-AFM and automated structure detection has been able to resolve the orientation of small organic 3D molecules from NC-AFM data [10]. Adapting these approaches to a broad range of $3 \mathrm{D}$ molecules bears the strong potential not only to gain insight into the on-surface adsorption properties of molecules, but also to unravel the intramolecular geometry. 
A prototypical class of molecules with a hindered rotation determines different conformers is the metallocene family. These molecules consist of a central metal atom sandwiched between two rotatable cyclopentadienyl $\left(\mathrm{C}_{5} \mathrm{H}_{5}, \mathrm{Cp}\right)$ rings. The limiting cases of the ring orientations are known as eclipsed and staggered conformers. For ferrocene, the rotational barrier between these conformers amounts to only $0.04 \mathrm{eV}$ in the gas phase [1113], while for substituted ferrocenes the energy barrier values determined in the gas phase amount up to $0.2 \mathrm{eV}$ [14-16]. In solution, barrier values of up to $0.7 \mathrm{eV}$ are measured $[17,18]$ and for ionised derivates calculations yielded rotational barrier values of up to $1.4 \mathrm{eV}$ [19].

From this class of molecules we investigate the ferrocene derivate 1,1'-ferrocene dicarboxylic acid (FDCA), a ferrocene functionalised with two carboxylic acid moieties. This molecule has been analysed before on $\mathrm{Ag}(111), \mathrm{Cu}(110)$, and $\mathrm{Cu}_{3} \mathrm{~N} /$ $\mathrm{Cu}(110)$ surfaces [20] as well as on the insulator surfaces calcite(104) [21] and $\mathrm{CaF}_{2}$ (111) [22]. An eclipsed ferrocene conformation was found to be predominant on the metallic surfaces [20] and on calcite(104) [21]. On $\mathrm{CaF}_{2}$ (111) surfaces, density functional theory (DFT) calculations revealed an optimum geometry of the eclipsed conformer in a nearly upright-standing, slightly tilted fashion, as well as a staggered conformer in an energetically less favourable geometry with one rotated carboxylic acid moiety [22]. These geometries, referred to as "geo 1" and "geo 2", are depicted in Figure 1a and Figure 1b, respectively. The molecule-surface bond, with a calculated binding energy for geo 1 of $-1.74 \mathrm{eV}$, is surprisingly strong [22]. Due to the tilted molecular orientations, both geometries have two hydrogen atoms as the topmost unit protruding the surface. However, the orientation with respect to the $\mathrm{CaF}_{2}(111)$ surface lattice differs for the two geometries.

In this work we investigate the NC-AFM contrast formation of FDCA molecules on $\mathrm{CaF}_{2}$ (111) surfaces. A distinct dumbbell shape has been observed in both topography and constantheight imaging modes in low-temperature experiments ( 5 and $77 \mathrm{~K}$ ) with qPlus sensors as well as at room temperature using silicon cantilevers [22]. Although the NC-AFM tips were not functionalised, i.e., not specifically terminated with atoms or molecules for imaging, we find a very good agreement between the experimental data and probe particle model (PPM) [23] calculations using $\mathrm{CO}$ and Xe tips. Our analysis suggest that contrast formation is governed by repulsion between the tip and the hydrogen atoms serving as markers for the molecular conformation.

\section{Experimental}

Scanning probe microscopy experiments were performed on $\mathrm{CaF}_{2}(111)$ bulk and $\mathrm{CaF}_{2} / \mathrm{CaF}_{1} / \mathrm{Si}(111)$ thin film samples under ultrahigh vacuum conditions ( $p<5 \times 10^{-10}$ mbar) in two separate systems equipped with appropriate facilities for in situ sample preparation.

Bulk $\mathrm{CaF}_{2}$ crystals (Korth Kristalle, Altenholz, Germany) were cleaved in vacuum [24] after degassing the crystal and sample holder. To reduce residual charge on the surface [25], the sample was heated at about $330 \mathrm{~K}$ for one hour prior to the deposition of the ferrocene molecules and NC-AFM experiments. Thin $\mathrm{CaF}_{2}$ films were prepared by deposition of $\mathrm{CaF}_{2}$ (purity 99.9\%, Alfa Aesar, Kandel, Germany) from an EFM3T e-beam evaporator (Focus GmbH, Huenstetten, Germany) on freshly prepared $\mathrm{Si}(111)-(7 \times 7)$ surfaces held at about $930 \mathrm{~K}$. Silicon substrates were highly B-doped p-type $\mathrm{Si}(111)$ samples (Institute of Electronic Materials Technology, Warsaw, Poland) with the $(7 \times 7)$ reconstruction prepared by flash cycles. Further details on the thin film growth and properties can be found in [22,26-28].

Deposition of 1,1'-ferrocene dicarboxylic acid (purity $98 \%$, Alfa Aesar, Kandel, Germany) on samples held at room temperature was accomplished by sublimation from custom-built Knudsen cells heated to about $390 \mathrm{~K}$.

For bulk samples, the [11 $\overline{2}]$ surface direction was determined by cleaving the crystal after the NC-AFM experiments along a (111) plane other than the surface plane. For thin film samples, the orientation of the $\operatorname{Si}(111)-(7 \times 7)$ unit cell was measured by scanning tunnelling microscopy (STM) and the [11 $\overline{2}]$ direction was determined by considering the B-type epitaxy of the $\mathrm{CaF}_{2} / \mathrm{CaF}_{1} / \mathrm{Si}(111)$ thin films samples, see $[22,26]$ for further details.

STM and NC-AFM experiments were conducted at low temperatures ( 5 and $77 \mathrm{~K}$ ) in two separate systems. Experiments on bulk crystals were performed using an Omicron LT qPlus gen.III instrument (ScientaOmicron GmbH, Taunusstein, Germany), while experiments on thin films used a ScientaOmicron LT qPlus gen.II machine, both operated with a MATRIX controller. W tips attached to tuning fork sensors in qPlus configuration [29] were used in both systems. For the measurements on thin film samples, custom-built sensors were fabricated with a separate tunnelling current wire to exclude cross-talk between the NC-AFM and tunnelling current signals [30], while sensors as supplied by the manufacturer were used for measurements on bulk $\mathrm{CaF}_{2}$ samples. Sensors were excited to oscillation amplitudes between about 0.15 and $0.8 \mathrm{~nm}$. Both instruments were equipped with an atom-tracking system for drift measurement and compensation [31]. All experimental data were analysed using Gwyddion [32]. 
The probe particle model (PPM) [23] was used for simulating NC-AFM imaging contrast. Neutral oxygen and xenon atoms were used as probe particles and frequency-shift $\Delta f$ data were calculated for an oscillation amplitude of $0.5 \mathrm{~nm}$. Lateral and vertical stiffness were chosen as 0.5 and $20 \mathrm{~N} / \mathrm{m}$, respectively. FDCA molecular models in the DFT-optimised geometries (using geo 1 and geo 2 from [22], see Figure 1a,b) were arranged along the $\mathrm{CaF}_{2}[11 \overline{2}]$ direction, resembling one molecular row of the $(2 \sqrt{2} \times \sqrt{2}) \mathrm{R} 45^{\circ}$ superstructure that was observed in the experiments. All simulated $\Delta f$ data are shown with a colour code representing a strong attractive tip-surface interaction as bright and a weak attractive or repulsive interaction as dark to allow for a direct comparison with the experimental data. We refer to this representation as "inverted colour scale".

\section{Results and Discussion}

Figure 1c and Figure 1d show examples of the prevalently observed dumbbell-shaped appearance of FDCA molecules on $\mathrm{CaF}_{2}$ (111) surfaces [22] for NC-AFM measurements performed at 5 and $77 \mathrm{~K}$ on bulk and thin film samples, respectively. Previously, no difference in the molecular properties when comparing adsorption on $\mathrm{CaF}_{2}(111)$ bulk and thin film surfaces has been observed [22].

The distance-dependency of the imaging contrast was analysed for constant-height data where frequency-shift images were acquired at different tip-sample distances, see Figure $2 b-e$. Data were acquired above a region where several FDCA molecules were arranged along the $[11 \overline{2}]$ direction (see STM data in Figure 2a), with a molecular separation determined by the $\mathrm{CaF}_{2}$ (111) lattice periodicity of $669 \mathrm{pm}$. Upon step-wise reducing the tip-surface distance, the experimental NC-AFM contrast evolves from an elliptic shape caused by attractive tip-sample interactions (Figure 2b) into ring-like structures, followed by the dumbbell shape (Figure 2c). Further reduction of the tip-sample distance then results in the evolution of a sharp line (one example marked by an arrow in Figure 2d), which suggests a relaxation of the front tip apex in agreement with earlier observations in organic [33,34] or inorganic [35,36] systems. For the smallest tip-sample separation, two short elongated dark segments (see markers in Figure 2e) evolve on one side of a sharp line connecting the weights of the dumbbells.

For an interpretation of the NC-AFM contrast, we run simulations based on the probe particle model [23] for three molecules aligned along $[11 \overline{2}]$, each molecule being in the DFToptimised adsorption geometry geo 1 [22], as shown in Figure 2o,p. We use Lennard-Jones parameters for CO and Xe tips, a neutral probe particle, and stiffness values of $\left(k_{x}, k_{y}, k_{z}\right)=$ $(0.5,0.5,20) \mathrm{N} / \mathrm{m}$, yielding the results for CO reproduced in Figure $2 \mathrm{f}-\mathrm{i}$ and for $\mathrm{Xe}$ in Figure $2 \mathrm{k}-\mathrm{n}$ at the same heights. Both
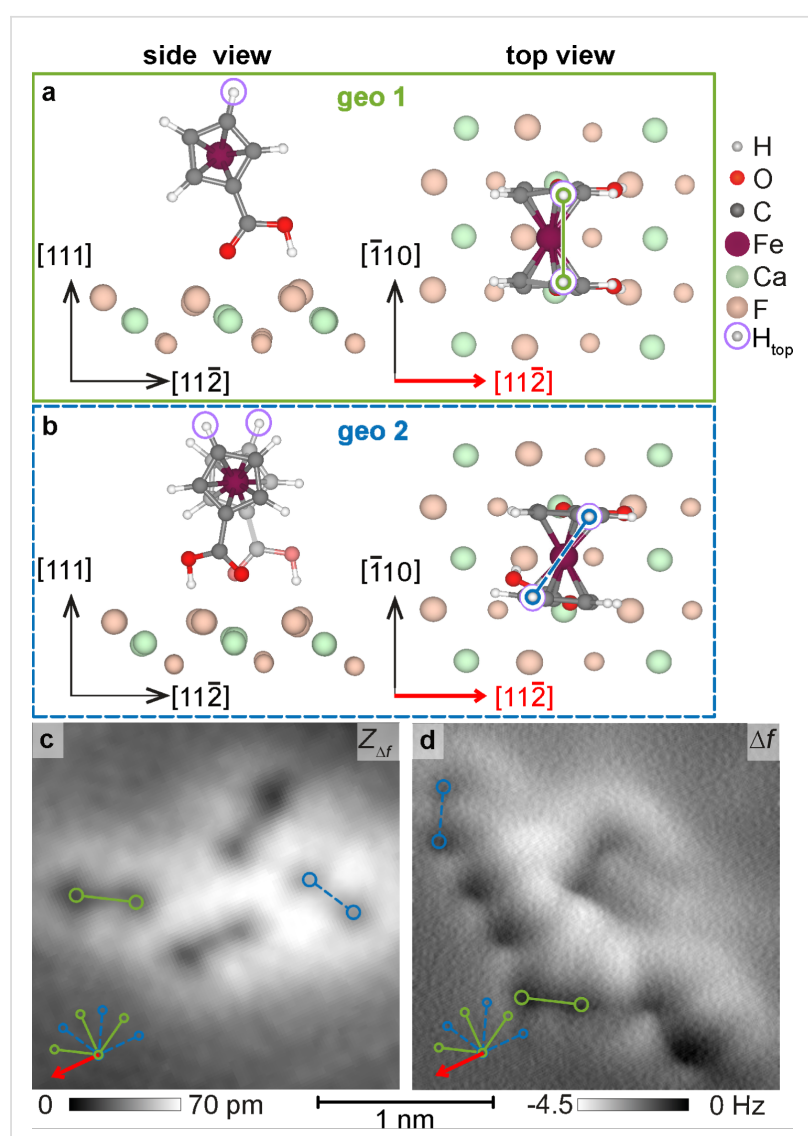

Figure 1: Dumbbell shape of single FDCA molecules. Quadruped binding motif of FDCA on $\mathrm{CaF}_{2}$ (111) (adapted from [22]) of (a) geo 1 and (b) geo 2. Dumbbell shape of FDCA molecules (c) on bulk $\mathrm{CaF}_{2}(111)$ measured by NC-AFM in topography mode and (d) on thin film $\mathrm{CaF}_{2}(111)$ surface measured in the constant-height mode (adapted from [22]). The orientations of the two geometries geo 1 and geo 2 are marked by solid green and dashed blue lines, respectively, and are included in all images with the respective orientation. (A five pixel averaging filter was applied to reduce noise within the frequency shift image. An inverted colour scale is used for the constant-height $\Delta f$ NC-AFM data to match the topography appearance.)

series of images exhibit a remarkable similarity to the experimental data. The change from the ellipse to the dumbbell shape is reproduced as well as the evolution of a sharp line connecting the two dumbbell weights (Figure 2h,1). The characteristic contrast features appear in the simulations at different heights for the different tips, a finding which we attribute to the different van der Waals radii of $\mathrm{CO}$ and xenon. From a comparison with the molecular geometry we can confidentially assign the dark weights of the dumbbell to repulsive interactions with the top hydrogen atoms $\mathrm{H}_{\text {top }}$ (see Figure 1a). The bright halo around these two weights is in turn caused by attractive forces acting between the tip and other atoms of the FDCA molecule. Thus, the PPM simulations allow for the assignment of the dumbbell shape to a single FDCA molecule and, in turn, the orientation of the dumbbell precisely defines the axis between the top hydrogen atoms. Additionally, the asymmetry that evolves 

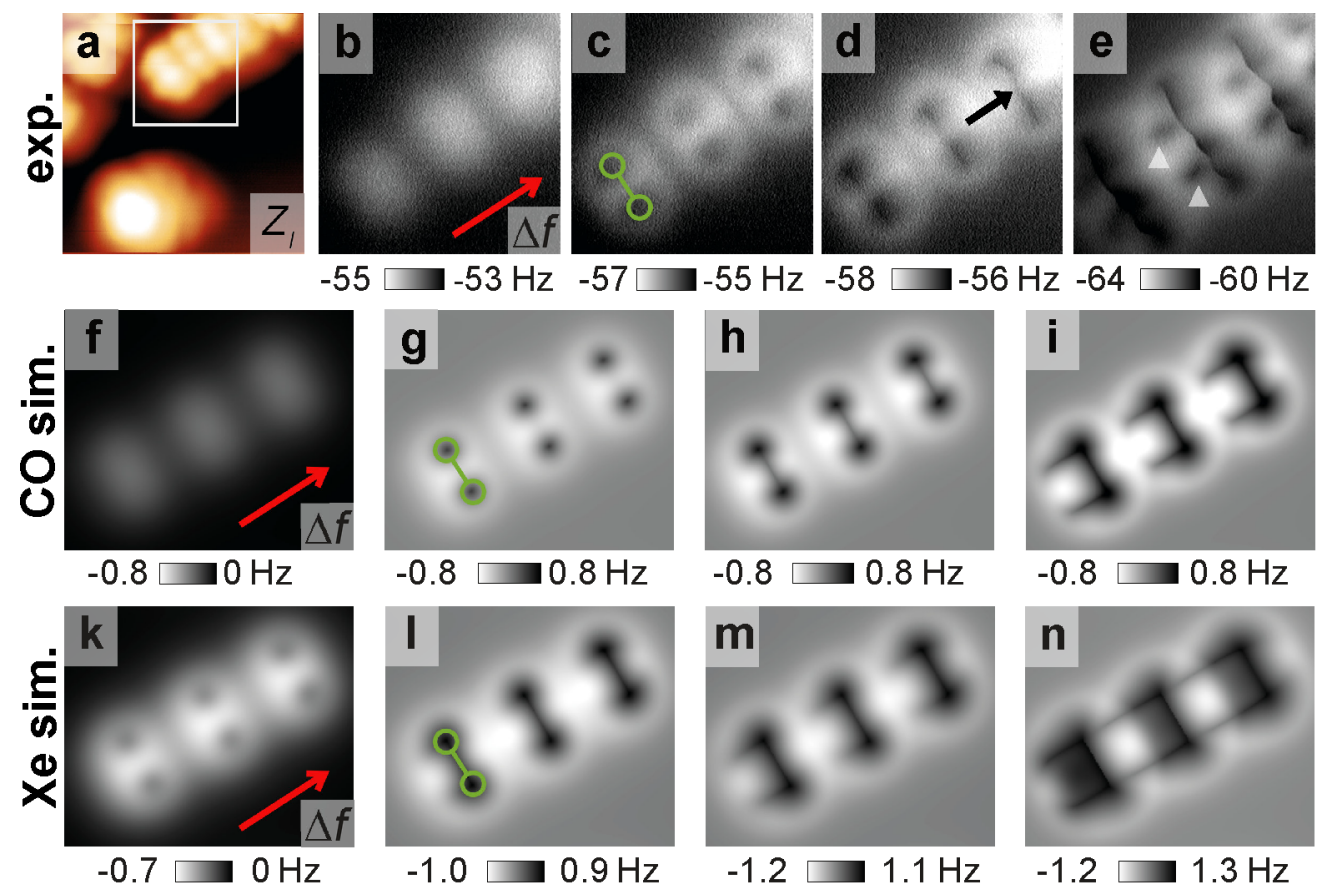

$-0.7 \square 0 \mathrm{~Hz}$

$-1.2$

$1.1 \mathrm{~Hz}$

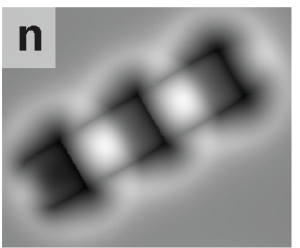

$-1.2 \square 1.3 \mathrm{~Hz}$

$\mathbf{0}$

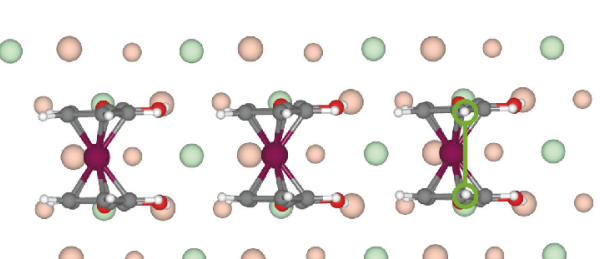

p
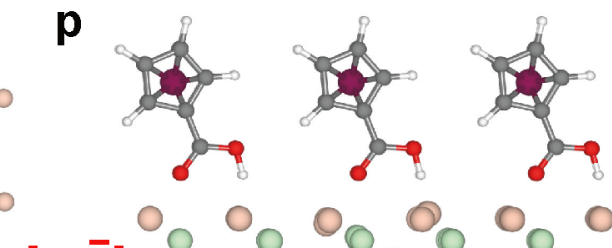

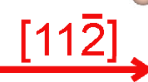

Figure 2: High-resolution NC-AFM imaging and simulation. Experimental and simulated frequency-shift images of a single molecular row along the [11ㄹ] direction (red arrows). Exemplary molecules are marked by a green dumbbell. (a) Experimental STM image $\left(U=4 \mathrm{~V}, l_{t}=2 \mathrm{pA}\right)$. (b-e) Experimental constant-height NC-AFM data $\left(f_{0}=24363 \mathrm{~Hz}, Q_{0} \approx 6000, A_{0}=0.27 \mathrm{~nm}\right)$. Frequency shift ranges are shown below each image (inverted colour scales are used). A four pixel averaging filter was applied to reduce noise within the frequency shift images. A slight tilt of the constant-height plane leads to a small modulation along the row. $(f-i)$ Simulated NC-AFM images using a CO tip model. $(k-n)$ Simulated NC-AFM images for a Xe tip model positioned with the tip backbone positioned at the same heights as for the $\mathrm{CO}$ image calculations. $(\mathrm{o}, \mathrm{p})$ Structure of the simulation cell with each molecule in the single-molecule DFT-optimised geometry geo 1 [22].

at small tip-sample distances is reproduced by the simulations, especially the two small, elongated dark segments marked by small triangles in Figure 2e. In the calculations, these dark segments are due to repulsive interactions with the lower-lying hydrogen atoms of the $\mathrm{Cp}$ rings, similar to the lines across the top bond of the $\mathrm{Cp}$ ring observed in previous measurements of FDCA on $\mathrm{Cu}(110)$ and $\mathrm{Cu}_{3} \mathrm{~N} / \mathrm{Cu}(110)$ surfaces [20]. We note that the location of these dark segments is here linked to the surface orientation by the molecular geometry, where the expected match with the experimental results is fulfilled.

The good match between the key features in experiments and calculations is remarkable as measurements were not performed with a specifically prepared tip. The accidental functionalisation of the tip might have occurred through the adsorption of a FDCA molecule to the tip with the carbonyl moiety facing the surface. This would be in line with earlier NC-AFM experiments of naphthalene tetracarboxylic diimide (NTCDI) adsorbed on Ag-terminated silicon surfaces [37]. In the latter case, the observation of submolecular contrast similar to images acquired with CO-terminated tips has been explained by a tipadsorbed NTCDI molecule with a CO-like carboxylic oxygen pointing towards the sample [37]. In the present experiments, a similar mechanism with one of the FDCA carbonyl groups pointing towards the sample is plausible. However, the agreement between experiment and simulation might also reflect a more general feature of repulsive mode imaging. While the tip-surface interaction force curves in the attractive region differ significantly from each other for different tip-terminating species, the force curves in the repulsive region are so steep that differences in their details have only minor influence on the NC-AFM contrast. Hence, the NC-AFM contrast observed for 
imaging with different tip terminations differs only by the distance of the onset of certain features as found for the simulations with $\mathrm{CO}$ and xenon tips. In either case, the interaction with the top hydrogen atoms is found to be localised and repulsive and serves as a marker for the hydrogen atom position.

A previous analysis of the molecular orientations highlighted the presence of a minority population of FDCA molecules exhibiting the adsorption geometry geo 2 with a partly staggered molecular conformation [22]. The protruding hydrogen atoms align in this geometry along a different direction with respect to geo 1 due to the partly staggered conformation of the $\mathrm{Cp}$ rings. The corresponding PPM image calculations with identical simulation parameters as before for a $\mathrm{CO}$ tip are shown in Figure 3 Although the molecular row still proceeds along the $[11 \overline{2}]$ direction, the orientation of the dumbbell shape differs. The angle between the dumbbell-connecting line and the [11 $\overline{2}]$ direction reduces from $90^{\circ}$ (geo 1 in Figure 2) to $60^{\circ}$ (geo 2 in Figure 3 ). This is a direct consequence of the partly staggered conformation, which moves the top hydrogen atoms to these positions. At further reduced distances (see Figure 3c), lines both along the position of the top $\mathrm{Cp}$ bonds and along the iron centre of the FDCA molecule are again revealed.

Experimental examples for the different orientations are given in Figure 1c and Figure 1d. Due to the three-fold rotational symmetry of the $\mathrm{CaF}_{2}(111)$ surface, a total of six orientations, three for each geometry, are possible and included as sketches in the lower left corners with solid green and dashed blue lines marking the orientation of geo 1 and geo 2, respectively. Molecules of both geometries oriented along one of the possible angles are present in the experimental images, two examples for each geometry are marked. The experimental contrast is similar to the pattern of a single molecule within the calculated images in Figure $2 \mathrm{~h}$ and Figure $2 \mathrm{~m}$ for geo 1 and Figure $3 \mathrm{~b}$ for geo 2.

\section{Conclusion}

We have investigated the origin of the dumbbell shape, which is observed when imaging FDCA molecules on $\mathrm{CaF}_{2}(111)$ surfaces with NC-AFM. Based on a comparison of experimental constant-height frequency-shift data with image calculations using the probe particle model, we identify repulsive interactions between the tip and the topmost hydrogen atoms of the $\mathrm{Cp}$ rings of single FDCA molecules as the fundamental cause for the dumbbell shape. Due to the strong molecule-surface bond, molecules are usually not manipulated while scanning in this repulsive mode. We found tips prepared by standard procedures to be mostly stable and sharp enough to locally enter the repulsive regime. Following the vision of [9], the measurement of the two hydrogen atom positions with respect to the orientation of the $\mathrm{CaF}_{2}$ (111) lattice allows us to identify the ferrocene conformer and the molecular orientation on the surface. The experimental observation of the dumbbell shape suggests that the prerequisite of a stable tip during repulsive interactions with the hydrogen atoms can easily be fulfilled, even at room temperature. This is in line with previous findings of stable tips for submolecular resolution imaging at $77 \mathrm{~K}[37,38]$ and $300 \mathrm{~K}[39]$.
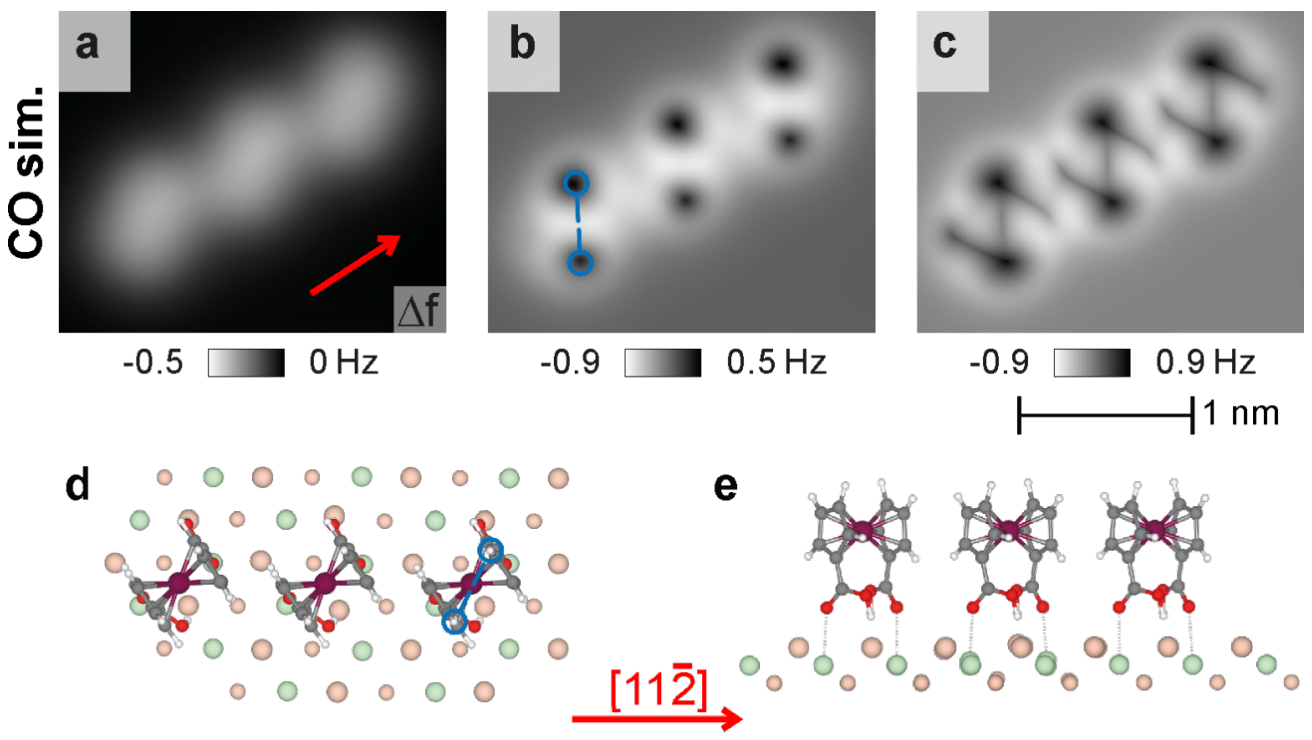

Figure 3: NC-AFM image calculation for a [11ㄹ] row of FDCA molecules in geo 2. (a-c) Constant-height frequency-shift $\Delta f$ data (inverted colour scale used) simulated at decreasing tip-sample distances for a row of FDCA molecules, each in geo 2 . The red arrows mark the [11 $\overline{2}$ ] direction and the dashed blue line (forming an angle of $60^{\circ}$ with the [11 2 ] direction) the molecular orientation for geo 2. (d) Top view and (e) side view of the simulation cell using geo 2 from [22]. 
Due to the predominant repulsive interactions with the chemically rather inert hydrogen atoms, we speculate that the chemical identity of the tip is therefore of secondary importance. Still, the tip has to be sharp enough to allow for resolving the dumbbell shape.

This is a demonstration for identifying hydrogen atoms in a 3D molecule with nonfunctionalised tips as well as the use of hydrogen atoms as markers for the molecular geometry. As the method introduced here is based on an universal imaging mechanism and widely applicable experimental procedures, we expect that the introduced method will allow for the identification of other conformers and, in particular, give further insights into adsorption geometries of complex 3D molecules.

The work further underlines the general applicability of repulsive-mode imaging of molecules independent of a specific tip termination, such as $\mathrm{CO}$, which is hitherto the standard tip for this imaging mode. We propose that high-resolution repulsivemode imaging of hydrogen atoms is possible with a variety of tips if the following three preconditions are fulfilled: (1) The molecule is sufficiently strongly bonded to the substrate to withstand manipulation due to lateral forces exerted by the approaching tip. (2) The tip atomic arrangement is characterised by a deep minimum of total energy preventing a rearrangement of tip atoms or disintegration if the tip is strained by the interaction with the molecule. (3) The tip end is atomically sharp. The third condition implies the stabilisation of the tipterminating atom by one or very few bonds directed towards the tip apex. Such a bond will always exhibit sufficient lateral elasticity to yield a sharp contrast feature when the tip terminating atom passes a molecular protrusion.

\section{Acknowledgements}

We are indebted to Philip J. Moriarty (University of Nottingham, UK) for supporting part of the experiments and for comments on the manuscript.

\section{Funding}

Financial support by the German Research Foundation (DFG) via grant RA2832/1-1 and from the People Programme (Marie Curie Actions) of the European Union's Seventh Framework Programme (FP7/2007-2013) under Research Executive Agency (REA) Grant No. 628439 are gratefully acknowledged. Computing time was granted by the University of Osnabrück via DFG project 239246210.

\section{ORCID ${ }^{\circledR}$ iDs}

Michael Reichling - https://orcid.org/0000-0003-3186-9000 Philipp Rahe - https://orcid.org/0000-0002-2768-8381

\section{References}

1. Woodruff, D. P. Rep. Prog. Phys. 2005, 68, 743-798. doi:10.1088/0034-4885/68/4/r01

2. Woodruff, D. P. J. Electron Spectrosc. Relat. Phenom. 2002, 126, 55-65. doi:10.1016/s0368-2048(02)00205-0

3. Hauschild, A.; Temirov, R.; Soubatch, S.; Bauer, O.; Schöll, A.; Cowie, B. C. C.; Lee, T.-L.; Tautz, F. S.; Sokolowski, M. Phys. Rev. B 2010, 81, 125432. doi:10.1103/physrevb.81.125432

4. Barth, C.; Foster, A. S.; Henry, C. R.; Shluger, A. L. Adv. Mater. (Weinheim, Ger.) 2011, 23, 477-501. doi:10.1002/adma.201002270

5. Hoffmann-Vogel, R. Rep. Prog. Phys. 2018, 81, 016501. doi:10.1088/1361-6633/aa8fda

6. Rahe, P.; Kittelmann, M.; Neff, J. L.; Nimmrich, M.; Reichling, M.; Maass, P.; Kühnle, A. Adv. Mater. (Weinheim, Ger.) 2013, 25 , 3948-3956. doi:10.1002/adma.201300604

7. Pavliček, N.; Fleury, B.; Neu, M.; Niedenführ, J.; Herranz-Lancho, C.; Ruben, M.; Repp, J. Phys. Rev. Lett. 2012, 108, 086101. doi:10.1103/physrevlett.108.086101

8. Pavliček, N.; Herranz-Lancho, C.; Fleury, B.; Neu, M.; Niedenführ, J.; Ruben, M.; Repp, J. Phys. Status Solidi B 2013, 250, 2424-2430. doi:10.1002/pssb.201349229

9. Kawai, S.; Nishiuchi, T.; Kodama, T.; Spijker, P.; Pawlak, R.; Meier, T.; Tracey, J.; Kubo, T.; Meyer, E.; Foster, A. S. Sci. Adv. 2017, 3, e1603258. doi:10.1126/sciadv.1603258

10. Alldritt, B.; Hapala, P.; Oinonen, N.; Urtev, F.; Krejci, O.; Federici Canova, F.; Kannala, J.; Schulz, F.; Liljeroth, P.; Foster, A. S. Sci. Adv. 2020, 6, eaay6913. doi:10.1126/sciadv.aay6913

11. Bohn, R. K.; Haaland, A. J. Organomet. Chem. 1966, 5, 470-476. doi:10.1016/s0022-328x(00)82382-7

12. Haaland, A.; Nilsson, J. E.; Olson, T.; Norin, T. Acta Chem. Scand. 1968, 22, 2653-2670. doi:10.3891/acta.chem.scand.22-2653

13. Coriani, S.; Haaland, A.; Helgaker, T.; Jørgensen, P. ChemPhysChem 2006, 7, 245-249. doi:10.1002/cphc.200500339

14. Sorriso, S. J. Organomet. Chem. 1979, 179, 205-213. doi:10.1016/s0022-328x(00)95223-9

15. Nowik, I.; Herber, R. H. Inorg. Chim. Acta 2000, 310, 191-195. doi:10.1016/s0020-1693(00)00295-4

16. Almenningen, A.; Haaland, A.; Samdal, S.; Brunvoll, J.; Robbins, J. L.; Smart, J. C. J. Organomet. Chem. 1979, 173, 293-299. doi:10.1016/s0022-328x(00)84784-1

17. McCulloch, B.; Brubaker, C. H., Jr. Organometallics 1984, 3 , 1707-1711. doi:10.1021/om00089a018

18. Shen, L.-H.; Okoroafor, M. O.; Brubaker, C. H., Jr. Organometallics 1988, 7, 825-829. doi:10.1021/om00094a006

19. Wang, X.-B.; Dai, B.; Woo, H.-K.; Wang, L.-S. Angew. Chem., Int. Ed. 2005, 44, 6022-6024. doi:10.1002/anie.200501564

20. Berger, J.; Kośmider, K.; Stetsovych, O.; Vondráček, M.; Hapala, P.; Spadafora, E. J.; Švec, M.; Jelínek, P. J. Phys. Chem. C 2016, 120, 21955-21961. doi:10.1021/acs.jpcc.6b05978

21. Rahe, P.; Steele, R. P.; Williams, C. C. Nano Lett. 2016, 16, 911-916. doi:10.1021/acs.nanolett.5b03725

22. Laflör, L.; Schlage, F. A.; Kantorovich, L.; Moriarty, P. J.; Reichling, M.; Rahe, P. J. Phys. Chem. C 2020, 124, 9900-9907. doi:10.1021/acs.jpcc.0c00115

23. Hapala, P.; Kichin, G.; Wagner, C.; Tautz, F. S.; Temirov, R.; Jelínek, P. Phys. Rev. B 2014, 90, 085421. doi:10.1103/physrevb.90.085421 
24. Tröger, L.; Schütte, J.; Ostendorf, F.; Kühnle, A.; Reichling, M. Rev. Sci. Instrum. 2009, 80, 063703. doi:10.1063/1.3152367

25. Barth, C.; Pakarinen, O. H.; Foster, A. S.; Henry, C. R. Nanotechnology 2006, 17, S128-S136. doi:10.1088/0957-4484/17/7/s05

26. Rahe, P.; Smith, E. F.; Wollschläger, J.; Moriarty, P. J. Phys. Rev. B 2018, 97, 125418. doi:10.1103/physrevb.97.125418

27. Olmstead, M. A. Heteroepitaxy of Disparate Materials: From Chemisorption to Epitaxy in $\mathrm{CaF}_{2} / \mathrm{Si}(111)$. In Thin Films: Heteroepitaxial Systems; Liu, W. K.; Santos, M. B., Eds.; World Scientific: Singapore, 1999; Vol. 15, pp 211-266. doi:10.1142/9789812816511_0005

28. Wollschläger, J. Resonant Tunneling Devices Based on Epitaxial Insulator-Semiconductor Structures: Growth and Characterisation of $\mathrm{CaF}_{2}$ Films on $\mathrm{Si}(111)$. In Recent Research Developments in Applied Physics; Pandalai, S., Ed.; Transworld Research Network: Trivandrum, India, 2002; Vol. 5-II, pp 621-695.

29. Giessibl, F. J. Appl. Phys. Lett. 1998, 73, 3956-3958. doi:10.1063/1.122948

30. Majzik, Z.; Setvín, M.; Bettac, A.; Feltz, A.; Cháb, V.; Jelínek, P. Beilstein J. Nanotechnol. 2012, 3, 249-259. doi:10.3762/bjnano.3.28

31. Rahe, P.; Schütte, J.; Schniederberend, W.; Reichling, M.; Abe, M.; Sugimoto, Y.; Kühnle, A. Rev. Sci. Instrum. 2011, 82, 063704. doi:10.1063/1.3600453

32. Nečas, D.; Klapetek, P. Cent. Eur. J. Phys. 2011, 10, 181-188. doi:10.2478/s11534-011-0096-2

33. Hämäläinen, S. K.; van der Heijden, N.; van der Lit, J.; den Hartog, S.; Liljeroth, P.; Swart, I. Phys. Rev. Lett. 2014, 113, 186102. doi:10.1103/physrevlett.113.186102

34. Peng, J.; Guo, J.; Hapala, P.; Cao, D.; Ma, R.; Cheng, B.; Xu, L.; Ondráček, M.; Jelínek, P.; Wang, E.; Jiang, Y. Nat. Commun. 2018, 9, 122. doi:10.1038/s41467-017-02635-5

35. Labidi, H.; Koleini, M.; Huff, T.; Salomons, M.; Cloutier, M.; Pitters, J.; Wolkow, R. A. Nat. Commun. 2017, 8, 14222. doi:10.1038/ncomms 14222

36. Sweetman, A.; Jarvis, S. P.; Rashid, M. A. Beilstein J. Nanotechnol. 2016, 7, 937-945. doi:10.3762/bjnano.7.85

37. Sweetman, A. M.; Jarvis, S. P.; Sang, H.; Lekkas, I.; Rahe, P.; Wang, Y.; Wang, J.; Champness, N. R.; Kantorovich, L.; Moriarty, P. Nat. Commun. 2014, 5, 3931. doi:10.1038/ncomms4931

38. Yesilpinar, D.; Schulze Lammers, B.; Timmer, A.; Amirjalayer, S.; Fuchs, H.; Mönig, H. Nanoscale 2020, 12, 2961-2965. doi:10.1039/c9nr10450j

39. Iwata, K.; Yamazaki, S.; Mutombo, P.; Hapala, P.; Ondráček, M.; Jelínek, P.; Sugimoto, Y. Nat. Commun. 2015, 6, 7766. doi:10.1038/ncomms8766

\section{License and Terms}

This is an Open Access article under the terms of the Creative Commons Attribution License (https://creativecommons.org/licenses/by/4.0). Please note that the reuse, redistribution and reproduction in particular requires that the authors and source are credited.

The license is subject to the Beilstein Journal of Nanotechnology terms and conditions: (https://www.beilstein-journals.org/bjnano)

The definitive version of this article is the electronic one which can be found at: https://doi.org/10.3762/bjnano.11.127 